\title{
INFLUENCE OF ORGANIC MANURES ON DROUGHT STRESS AT DIFFERENT GROWTH STAGES OF WHEAT
}

\author{
A.K.M.R. Amin and M.A. Baque \\ Department of Agronomy, Sher-e-Bangla Agricultural University \\ Corresponding E-mail: ruhulsau@yahoo.com
}

(Received: 30 September, 2020, Accepted: 22 November, 2020)

Keywords: Organic manure, drought stress, yield, wheat

\begin{abstract}
A pot experiment was conducted in the net house of the Agronomy department, Sher-eBangla Agricultural University, Dhaka during the period from November, 2018 to March, 2019 to evaluate the suitable organic source to mitigate the drought stress impact on wheat. The experiment comprised two factors viz. factor A: Three levels of organic manures, i) $\mathrm{O}_{0}=$ Control (Without organic manure), ii) $\mathrm{O}_{1=}$ Cowdung (10 t/ha), iii) $\mathrm{O}_{2}=$ Poultry litter ( $5 \mathrm{t} / \mathrm{ha}$ ), and factor $\mathrm{B}$ : four levels of drought i) $\mathrm{D}_{0}=$ Control (without drought), ii) $\mathrm{D}_{1}=$ Crown root initiation stage (at 20-29 DAS), iii) $\mathrm{D}_{2}=$ Booting stage (at 45-54 DAS), iv) $\mathrm{D}_{3}=$ Anthesis stage (at 55-64 DAS). The experiment was laid out in a completely randomized design (factorial) with three replications. Organic manure showed positive impact on yield and cowdung $\left(\mathrm{O}_{1}\right)$ gave the highest grain yield $\left(4.06 \mathrm{~g} \mathrm{plant}^{-1}\right)$. This may be attributed to the highest number of effective tillers plant ${ }^{-1}$ (3.21), spike length $(9.53 \mathrm{~cm})$, spikelet spike ${ }^{-1}(17.31)$, grains spikelet ${ }^{-1}(2.31)$ and 1000-grain weight (44.61 g) of wheat in this treatment. In respect of drought imposition treatments, grain yield was found higher in control treatment (without imposition of drought) which was statistically similar with drought imposition at booting stage treatment $\left(D_{2}\right)$. These two treatments also showed highest number of effective tillers plant ${ }^{-1}$, spikelets spike ${ }^{-1}$, grains spike ${ }^{-1}$ and 1000-grain weight. Regarding the interaction of organic manure and drought, cowdung without drought imposition $\left(\mathrm{O}_{1} \mathrm{D}_{0}\right)$ and cowdung with drought imposition at booting stage $\left(\mathrm{O}_{1} \mathrm{D}_{2}\right)$ were highest yielder among the other interactions which was attributed to higher 1000 -seed weight, number of effective tillers plant ${ }^{-1}$, spikelets spike ${ }^{-1}$ and grains spike ${ }^{-1}$. Results revealed that application of organic manure could reduce the impact of drought on wheat irrespective of growth stages. However, application of cowdung (10 t ha $\left.{ }^{-1}\right)$ was found more effective to combat drought impact at booting stage of wheat compared to other growth stages.
\end{abstract}

\section{Introduction}

Wheat (Triticum aestivum $L$ ) is the most important cereal crop in the world and it is one of the main sources of carbohydrate and also contains a considerable amount of protein, minerals and vitamins. It has significant role in human nutrition. Drought stress is one of the major abiotic stresses, which adversely affects crop growth and yield. Drought is the most common environmental stress affecting about $32 \%$ of the 99 million hectares under wheat cultivation in developing countries and at least 60 million hectares under wheat cultivation in developed countries (Rajaram, 2000). Drought stress reduces plant growth by affecting various physiological and biochemical processes, such as transpiration, translocation, ion uptake and nutrient metabolism (Farooq et al., 2008). Understanding of plant responses to drought stress is of great importance and a fundamental part for making the crops tolerant to stress conditions (Zhao et al., 2008). Some studies suggest that drought stress influences the thermal tolerance of photosynthesis (Havaux, 1992). Mirzaei et al. (2011) reported that drought stress induced at all growth stages reducing grain yield and yield components. Drought stress at stages of 
stem elongation, flowering and grain filling stages induced 32, 32 and $35 \%$ reduce in grain yield, respectively. Sarwar (2005) also found that grain yield and yield components of wheat significantly increased with the application of different organic materials resulting in the compost to be the most superior one. In addition, Yassen et al. (2006) was found that the irrigation at $60 \%$ water holding capacity and applying mineral nitrogen $60 \mathrm{~kg} / \mathrm{fed}$, with presence of the chicken manure as an organic fertilizer produced the highest wheat yield through two growth seasons. As such, this experiment was designed to determine the effect of water stress at different growth stages of wheat and to combat water stress through application of organic manure.

\section{Materials and Methods}

The pot experiment was conducted at the net house of the department of agronomy, Sher-e-Bangla Agricultural University (SAU), Dhaka-1207, during the period of November 2018 to March 2019. The experimental field was located at $23^{\circ} 41^{\prime} \mathrm{N}$ latitude and $90^{\circ} 22^{\prime}$ E longitude at a height of $8.6 \mathrm{~m}$ above the sea level. The soil of the experimental site was clay loam belonging to the "Madhupur Tract" under AEZ 28. The experiment comprised two factors viz. factor A: Organic manure $(\mathrm{O})-3, \mathrm{i}) \mathrm{O}_{0}=\mathrm{Control}$ (Recommended dose of chemical fertilizer/ RDCF + without organic fertilizer ), ii) $\mathrm{O}_{1}=\mathrm{RDCF}+$ Cowdung, iii) $\mathrm{O}_{2}=\mathrm{RDCF}+$ Poultry litter, factor $\mathrm{B}$ : Comprising drought by removing irrigation -4 i) $\mathrm{D}_{0}$ $=$ Control (without drought), ii) $\mathrm{D}_{1}=$ Crown root initiation stage (at 19-20 DAS), iii) $\mathrm{D}_{2}=$ Booting stage (at 45-54 DAS), iv) $\mathrm{D}_{3}=$ Anthesis stage (at 55-64 DAS). The experiment was laid out in a completely randomized design (factorial) with three replications. A total of 36 earthen pots measuring $22 \mathrm{~cm}$ diameter and $18 \mathrm{~cm}$ height were collected from the local market. Each pot was fill-up with $20 \mathrm{~kg}$ of soil. Urea, TSP, MoP, Gypsum, Zinc oxide and Boric acid were used at the rate of 200, 72, 66, 110, 4 and $5 \mathrm{~kg} \mathrm{ha}^{-1}\left(2.00,0.72,0.66,1.10,0.04\right.$ and $\left.0.05 \mathrm{~g} \mathrm{pot}^{-1}\right)$, respectively mixed with the soil before fill-up the pot except urea. Urea was applied in three equal splits at pot filling up, 21 DAS and 55 DAS. Cowdung and poultry litter were applied in the pots @ 10 and $5 \mathrm{t} \mathrm{ha}^{-1}$, respectively as per treatment. Seeds of wheat variety BARI Gom28 were collected from Wheat Research Center, Bangladesh Agriculture Research Institute (BARI) campus, Joydebpur, Gazipur. Before sowing, seeds were treated with Provex 200EC @ $2.5 \mathrm{~g}$ powder for $\mathrm{kg}^{-1}$ seed. Ten seeds were sown in each pot on $23^{\text {rd }}$ November 2018. After sowing, the seeds were covered with soil and lightly pressed by hand. Five plants were kept for assessment in each pot after 14 DAS. Different intercultural operations were done to ensure normal growth of the crop except irrigation. Irrigation was applied as per need of treatment of the experiment where irrigation was not applied during drought imposition period(s) treatments. The crop was harvested at different dates on the basis of physiological maturity. The crop was harvested on 11 March, 2019. Data on different crop characters, yield attributes and yield were collected from the harvested five plants from each pot. Threshing, cleaning and drying of grains were done separately for each treatment. Properly dried grain and straw were weighed and converted into g plant ${ }^{-1}$ basis. The collected data of each pot were statistically analyzed to obtain the level of significance using the computer-based software Statistics10. Mean difference among the treatments were tested with Duncan's Multiple Range Test (DMRT) test at $5 \%$ level of significance.

\section{Results and Discussion}

Significant difference existed among different organic manures with respect to yield and yield contributing characters (Table 1 and Table 2). The yield advantages of $0.45 \mathrm{~g}$ and $0.75 \mathrm{~g}_{\text {plant }}{ }^{-1}$ for $\mathrm{O}_{1}$ (Cowdung) applied pot over $\mathrm{O}_{2}$ (poultry litter) and $\mathrm{O}_{0}$ (no organic manure) applied pot was found possibly aided by higher tillers plant ${ }^{-1}(3.21)$, spike length $(9.53 \mathrm{~cm})$, spikelets spike ${ }^{-1}(17.31)$, grains spike $^{-1}(2.31)$, weight of 1000 -grains (44.61 g), straw yield $\left(4.44 \mathrm{~g}^{-1 a n t^{-1}}\right)$, biological yield $(8.50 \mathrm{~g}$ plant $^{-1}$ ) and harvest index $(47.26 \%)$ in the $\mathrm{O}_{2}$ (Cowdung) applied treatment. The result agrees with the findings of Ibrahim et al. (2008) and Hammad et al. (2011) that organic fertilizer increased wheat yield 
over control. According to Uyanoz et al. (2006) yield attributes of wheat improve with organic manure which corroborates with the present findings.

Table 1. Effect of organic manures on plant height and yield attributes of wheat

\begin{tabular}{|c|c|c|c|c|c|c|c|}
\hline $\begin{array}{l}\text { Organic } \\
\text { manure }\end{array}$ & $\begin{array}{c}\text { Plant } \\
\text { height } \\
(\mathrm{cm})\end{array}$ & $\begin{array}{c}\text { Effective } \\
\text { tillers } \\
\text { plant }^{-1}(\text { no. })\end{array}$ & $\begin{array}{c}\text { Spike } \\
\text { length } \\
(\mathrm{cm})\end{array}$ & $\begin{array}{c}\text { Spikelet } \\
\text { Spike }^{-1} \\
\text { (no.) }\end{array}$ & $\begin{array}{c}\text { Grains } \\
\text { spikelet }^{-1} \\
\text { (no.) }\end{array}$ & $\begin{array}{c}\text { Grains } \\
\text { spike }^{-1} \\
\text { (no.) }\end{array}$ & $\begin{array}{l}\text { Weight of } \\
1000 \text { grains } \\
\text { (g) }\end{array}$ \\
\hline $\mathrm{O}_{0}$ & $69.105 \mathrm{~b}$ & $2.02 \mathrm{c}$ & $8.34 \mathrm{~b}$ & $16.18 \mathrm{~b}$ & $1.94 \mathrm{~b}$ & $31.01 \mathrm{c}$ & $39.69 \mathrm{~b}$ \\
\hline $\mathrm{O}_{1}$ & $76.779 \mathrm{a}$ & $3.21 \mathrm{a}$ & $9.53 \mathrm{a}$ & $17.31 \mathrm{a}$ & $2.31 \mathrm{a}$ & $37.16 \mathrm{a}$ & $44.61 \mathrm{a}$ \\
\hline $\mathrm{O}_{2}$ & $70.171 \mathrm{~b}$ & $2.65 \mathrm{~b}$ & $8.50 \mathrm{~b}$ & $16.52 \mathrm{~b}$ & $1.92 \mathrm{~b}$ & $32.71 \mathrm{~b}$ & $40.41 \mathrm{~b}$ \\
\hline SE & 2.42 & 0.14 & 0.19 & 0.33 & 0.06 & 0.70 & 0.99 \\
\hline CV (\%) & 8.23 & 13.17 & 5.21 & 4.90 & 7.60 & 5.09 & 5.86 \\
\hline
\end{tabular}

In a column means having similar letter(s) are statistically similar and those having dissimilar letter(s) differ significantly at $5 \%$ level of significance

Here: $\mathrm{O}_{0}=$ Control (Only RDCF + no organic manure), $\mathrm{O}_{1}=\mathrm{RDCF}+$ Cowdung, $\mathrm{O}_{2}=\mathrm{RDCF}+$ Poultry litter

Table 2. Effect of organic manure on yields and harvest index of wheat

\begin{tabular}{|c|c|c|c|c|}
\hline Organic manure & $\begin{array}{l}\text { Grain yield } \\
\text { plant }^{-1} \\
(\mathrm{~g})\end{array}$ & $\begin{array}{c}\text { Straw yield plant } \\
(\mathrm{g})\end{array}$ & $\begin{array}{l}\text { Biological } \\
\text { yield plant }{ }^{-1} \\
\text { (g) }\end{array}$ & $\begin{array}{c}\text { Harvest index } \\
(\%)\end{array}$ \\
\hline $\mathbf{O}_{0}$ & $3.31 \mathrm{c}$ & $3.73 \mathrm{c}$ & $7.04 \mathrm{c}$ & 46.66 \\
\hline$O_{1}$ & $4.06 \mathrm{a}$ & $4.44 \mathrm{a}$ & $8.50 \mathrm{a}$ & 47.26 \\
\hline $\mathrm{O}_{2}$ & $3.56 \mathrm{~b}$ & $4.05 \mathrm{~b}$ & $7.61 \mathrm{~b}$ & 46.51 \\
\hline SE & 0.0819 & 0.776 & 0.1526 & NS \\
\hline $\mathrm{CV}(\%)$ & 5.51 & 4.67 & 4.85 & 3.75 \\
\hline
\end{tabular}

In a column means having similar letter(s) are statistically similar and those having dissimilar letter(s) differ significantly at $5 \%$ level of significance

Here: $\mathrm{O}_{0}=$ Control (Only RDCF + no organic manure), $\mathrm{O}_{1}=\mathrm{RDCF}+$ Cowdung, $\mathrm{O}_{2}=\mathrm{RDCF}+$ Poultry litter

The result reveled that among the drought imposed treatments, the control plants $\mathrm{D}_{0}$ (without drought) and $\mathrm{D}_{2}$ (drought imposition at booting stage, 45-54 DAS) showed highest and statistically similar grain yield, effective tillers hill ${ }^{-1}$, spikelet spike $e^{-1}$, grains spike ${ }^{-1}$, weight of 1000 gains and harvest index (Table 3 and Table 4). $\mathrm{D}_{0}$ (without drought) treatment was out yielded by producing $28.99 \%$ and $65.78 \%$ higher yield over $\mathrm{D}_{1}$ (drought imposition at crown root initiation stage) and $\mathrm{D}_{3}$ (drought imposition at anthesis stage), respectively. On the other hand, $\mathrm{D}_{2}$ (drought imposition at booting stage) treatment was out yielded by producing $23.96 \%$ and $59.32 \%$ higher yield over $\mathrm{D}_{1}$ (drought imposition at crown root initiation stage) and $\mathrm{D}_{3}$ (drought imposition at anthesis stage), respectively. The treatment $\mathrm{D}_{0}$ (without drought) also produced highest level of tillers plant ${ }^{-1}$, spikelets spike ${ }^{-1}$, grains spike ${ }^{-1}$, straw yield, biological yield and harvest index than drought imposition plants. However, among the drought imposition treatments $\mathrm{D}_{2}$ (drought at booting stage) gave highest yield and yield attributes than other drought imposition treatments. The present result was supported by the findings of Akram (2011) that drought imposition at different growth stages caused severe reduction in yield and yield components of wheat. Similar result was also observed by Alghabari and Isham, (2018) that drought stress affected barley yield through impaired grain development and grain filling duration. 
Table 3. Effect of drought stress on plant height and yield attributes of wheat

\begin{tabular}{cccccccc}
\hline $\begin{array}{c}\text { Drought } \\
\text { stage }\end{array}$ & $\begin{array}{c}\text { Plant } \\
\text { height } \\
(\mathbf{c m})\end{array}$ & $\begin{array}{c}\text { Effective } \\
\text { tillers } \\
(\mathbf{n o .})\end{array}$ & $\begin{array}{c}\text { Spike } \\
\text { length } \\
(\mathbf{c m})\end{array}$ & $\begin{array}{c}\text { Spikelet } \\
\text { Spike }^{-\mathbf{1}} \\
(\mathbf{n o .})\end{array}$ & $\begin{array}{c}\text { Grains } \\
\text { Spikelet }^{-1} \\
(\mathbf{n o})\end{array}$ & $\begin{array}{c}\text { Grains } \\
\text { Spike }^{-\mathbf{1}} \\
(\mathbf{n o .})\end{array}$ & $\begin{array}{c}\text { Weight of } \\
\mathbf{1 0 0 0} \text { grain } \\
(\mathbf{g})\end{array}$ \\
\hline $\mathbf{D}_{\mathbf{0}}$ & $76.02 \mathrm{a}$ & $2.93 \mathrm{a}$ & $9.82 \mathrm{a}$ & $17.94 \mathrm{a}$ & $2.29 \mathrm{a}$ & $36.70 \mathrm{a}$ & $44.82 \mathrm{a}$ \\
$\mathbf{D}_{\mathbf{1}}$ & $69.82 \mathrm{bc}$ & $2.47 \mathrm{bc}$ & $8.40 \mathrm{c}$ & $16.00 \mathrm{~b}$ & $1.95 \mathrm{~b}$ & $32.16 \mathrm{~b}$ & $40.33 \mathrm{~b}$ \\
$\mathbf{D}_{\mathbf{2}}$ & $74.73 \mathrm{ab}$ & $2.75 \mathrm{ab}$ & $9.11 \mathrm{~b}$ & $17.62 \mathrm{a}$ & $2.16 \mathrm{a}$ & $35.26 \mathrm{a}$ & $43.10 \mathrm{a}$ \\
$\mathbf{D}_{\mathbf{3}}$ & $67.50 \mathrm{c}$ & $2.36 \mathrm{c}$ & $7.83 \mathrm{~d}$ & $15.10 \mathrm{c}$ & $1.83 \mathrm{~b}$ & $30.40 \mathrm{c}$ & $38.05 \mathrm{~b}$ \\
\hline $\mathrm{SE}$ & 2.7952 & 0.1630 & 0.2161 & 0.3852 & 0.0737 & 0.8072 & 1.14 .76 \\
$\mathrm{CV}(\%)$ & 8.23 & 13.17 & 5.21 & 4.90 & 7.60 & 5.09 & 5.86 \\
\hline
\end{tabular}

In a column means having similar letter(s) are statistically similar and those having dissimilar letter(s) differ significantly at $5 \%$ level of significance

Here: $\mathrm{D}_{0}=$ Control (No stress), $\mathrm{D}_{1}=$ Crown root initiation stage (20-19 DAS), $\mathrm{D}_{2}=$ Booting stage $(45-54$ DAS $)$ and $\mathrm{D}_{3}=$ Anthesis stage (55-64 DAS)

Table 4. Effect of drought stress on yields and harvest index of wheat

\begin{tabular}{ccccc}
\hline Drought stage & $\begin{array}{c}\text { Grain yield } \\
\text { plant }^{-1} \\
(\mathbf{g})\end{array}$ & $\begin{array}{c}\text { Straw yield } \\
\text { plant }^{-\mathbf{1}} \\
(\mathbf{g})\end{array}$ & $\begin{array}{c}\text { Biological } \\
\text { yield plant }^{\mathbf{1}} \\
(\mathbf{g})\end{array}$ & $\begin{array}{c}\text { Harvest index } \\
(\%)\end{array}$ \\
\hline $\mathrm{D}_{0}$ & $4.36 \mathrm{a}$ & $4.70 \mathrm{a}$ & $9.06 \mathrm{a}$ & $48.10 \mathrm{a}$ \\
$\mathrm{D}_{1}$ & $3.38 \mathrm{~b}$ & $3.89 \mathrm{c}$ & $7.27 \mathrm{c}$ & $46.29 \mathrm{~b}$ \\
$\mathrm{D}_{2}$ & $4.19 \mathrm{a}$ & $4.43 \mathrm{~b}$ & $8.62 \mathrm{~b}$ & $48.27 \mathrm{a}$ \\
$\mathrm{D}_{3}$ & $2.63 \mathrm{c}$ & $3.26 \mathrm{~d}$ & $5.90 \mathrm{~d}$ & $44.59 \mathrm{~b}$ \\
\hline $\mathrm{SE}$ & 0.0946 & 0.0896 & 0.1762 & 0.8268 \\
$\mathrm{CV}(\%)$ & 5.51 & 4.67 & 4.85 & 3.75 \\
\hline
\end{tabular}

In a column means having similar letter(s) are statistically similar and those having dissimilar letter(s) differ significantly at $5 \%$ level of significance

Here: $\mathrm{D}_{0}=$ Control (No stress), $\mathrm{D}_{1}=$ Crown root initiation stage (20-19 DAS), $\mathrm{D}_{2}=$ Booting stage (45-54 DAS)

and $\mathrm{D}_{3}=$ Anthesis stage (55-64 DAS)

Interaction of organic manure and drought stress treatment showed significant variation in all the studied parameters in this experiment (Table 5 and Table 6). The interaction of $\mathrm{O}_{1} \mathrm{D}_{0}$ (Cowdung $\times$ without drought treatment) and $\mathrm{O}_{1} \mathrm{D}_{2}$ (Cowdung $\times$ drought at booting stage) performed best in respect of grain yield (4.72 and $4.55 \mathrm{~g} \mathrm{plant}^{-1}$, respectively) which may be attributed to highest effective tillers plant $^{-1}$, spike length, spikelet spike ${ }^{1}$, grains spikelet ${ }^{1}$ and weight of 1000 -grain in these interactions.

Table 5. Interaction effects of organic manure and drought stress on plant height and yield contributing characters of wheat

\begin{tabular}{|c|c|c|c|c|c|c|c|}
\hline $\begin{array}{c}\text { Interaction } \\
\text { (Organic } \\
\text { manu. } \times \\
\text { Drou.) }\end{array}$ & $\begin{array}{c}\text { Plant } \\
\text { height } \\
(\mathrm{cm})\end{array}$ & $\begin{array}{c}\text { Effective } \\
\text { tillers } \\
\text { plant }^{-1} \\
\text { (no.) }\end{array}$ & $\begin{array}{c}\text { Spike } \\
\text { length } \\
(\mathrm{cm})\end{array}$ & $\begin{array}{c}\text { Spike let } \\
\text { spike }^{-1} \\
\text { (no.) }\end{array}$ & $\begin{array}{c}\text { Grains } \\
\text { spikelet }^{-1} \\
\text { (no.) }\end{array}$ & $\begin{array}{c}\text { Grains } \\
\text { spike }^{-1} \\
\text { (no.) }\end{array}$ & $\begin{array}{c}\text { Weight of } \\
\text { 1000- } \\
\text { grains } \\
\text { (g) }\end{array}$ \\
\hline$\overline{\mathrm{O}_{0} \mathrm{D}_{0}}$ & 70.76 a-d & $2.21 \mathrm{e}$ & $9.44 \mathrm{bc}$ & $17.18 \mathrm{bc}$ & $2.15 \mathrm{bc}$ & $34.09 \mathrm{bc}$ & $45.14 \mathrm{a}-\mathrm{c}$ \\
\hline $\mathrm{O}_{0} \mathrm{D}_{1}$ & $68.40 \mathrm{~b}-\mathrm{d}$ & $1.88 \mathrm{e}$ & $7.87 \mathrm{f}-\mathrm{h}$ & $15.61 \mathrm{~d}-\mathrm{f}$ & $1.82 \mathrm{ef}$ & $29.56 \mathrm{ef}$ & $37.76 \mathrm{fg}$ \\
\hline $\mathrm{O}_{0} \mathrm{D}_{2}$ & $71.53 \mathrm{a}-\mathrm{d}$ & $2.10 \mathrm{e}$ & $8.77 \mathrm{c}-\mathrm{e}$ & $17.61 \mathrm{ab}$ & $1.87 \mathrm{de}$ & $32.22 \mathrm{c}-\mathrm{e}$ & $41.91 \mathrm{~b}-\mathrm{e}$ \\
\hline $\mathrm{O}_{0} \mathrm{D}_{3}$ & $65.73 \mathrm{~cd}$ & $1.87 \mathrm{e}$ & $7.27 \mathrm{~h}$ & $14.29 \mathrm{f}$ & $1.93 \mathrm{c}-\mathrm{e}$ & $28.19 \mathrm{f}$ & $33.97 \mathrm{~g}$ \\
\hline $\mathrm{O}_{1} \mathrm{D}_{0}$ & $79.83 \mathrm{a}$ & $3.48 \mathrm{a}$ & $10.79 \mathrm{a}$ & $18.91 \mathrm{a}$ & $2.51 \mathrm{a}$ & $40.02 \mathrm{a}$ & $46.99 \mathrm{a}$ \\
\hline $\mathrm{O}_{1} \mathrm{D}_{1}$ & $75.47 \mathrm{a}-\mathrm{c}$ & $3.12 \mathrm{ab}$ & $9.08 \mathrm{~b}-\mathrm{d}$ & $16.56 \mathrm{~b}-\mathrm{d}$ & $2.13 \mathrm{~b}-\mathrm{d}$ & $35.83 \mathrm{~b}$ & $43.28 \mathrm{a}-\mathrm{d}$ \\
\hline $\mathrm{O}_{1} \mathrm{D}_{2}$ & $78.72 \mathrm{a}-\mathrm{d}$ & $3.32 \mathrm{ab}$ & $9.72 \mathrm{~b}$ & $17.72 \mathrm{ab}$ & $2.63 \mathrm{a}$ & $39.02 \mathrm{a}$ & $45.93 \mathrm{ab}$ \\
\hline
\end{tabular}




\begin{tabular}{cccccccc}
$\mathrm{O}_{1} \mathrm{D}_{3}$ & $73.10 \mathrm{a}-\mathrm{d}$ & $2.95 \mathrm{a}-\mathrm{c}$ & $8.54 \mathrm{~d}-\mathrm{f}$ & $16.03 \mathrm{c}-\mathrm{e}$ & $1.99 \mathrm{~b}-\mathrm{e}$ & $33.78 \mathrm{~b}-\mathrm{d}$ & $42.25 \mathrm{~b}-\mathrm{d}$ \\
$\mathrm{O}_{2} \mathrm{D}_{0}$ & $77.47 \mathrm{ab}$ & $3.10 \mathrm{ab}$ & $9.22 \mathrm{~b}-\mathrm{d}$ & $17.73 \mathrm{ab}$ & $2.20 \mathrm{~b}$ & $36.00 \mathrm{~b}$ & $42.32 \mathrm{~b}-\mathrm{d}$ \\
$\mathrm{O}_{2} \mathrm{D}_{1}$ & $65.60 \mathrm{~cd}$ & $2.40 \mathrm{c}-\mathrm{e}$ & $8.24 \mathrm{e}-\mathrm{g}$ & $15.83 \mathrm{c}-\mathrm{e}$ & $1.91 \mathrm{c}-\mathrm{e}$ & $31.08 \mathrm{~d}-\mathrm{f}$ & $39.95 \mathrm{~d}-\mathrm{f}$ \\
$\mathrm{O}_{2} \mathrm{D}_{2}$ & $73.95 \mathrm{a}-\mathrm{c}$ & $2.83 \mathrm{~b}-\mathrm{d}$ & $8.84 \mathrm{c}-\mathrm{e}$ & $17.53 \mathrm{ab}$ & $1.98 \mathrm{~b}-\mathrm{e}$ & $34.53 \mathrm{bc}$ & $41.46 \mathrm{c}-\mathrm{f}$ \\
$\mathrm{O}_{2} \mathrm{D}_{3}$ & $63.67 \mathrm{~d}$ & $2.26 \mathrm{de}$ & $7.69 \mathrm{gh}$ & $14.97 \mathrm{ef}$ & $1.57 \mathrm{f}$ & $29.22 \mathrm{f}$ & $37.92 \mathrm{e}-\mathrm{g}$ \\
\hline $\mathrm{SE}$ & 4.84 & 0.28 & 0.37 & 0.66 & 0.12 & 1.39 & 1.98 \\
$\mathrm{CV}(\%)$ & 8.23 & 13.17 & 5.21 & 4.90 & 7.60 & 5.09 & 5.86 \\
\hline
\end{tabular}

In a column means having similar letter(s) are statistically similar and those having dissimilar letter(s) differ significantly at $5 \%$ level of significance

Here: $\mathrm{O}_{0}=$ Control (Only RDCF+ no organic manure), $\mathrm{O}_{1}=\mathrm{RDCF}+$ Cowdung, $\mathrm{O}_{2}=\mathrm{RDCF}+$ Poultry, $\mathrm{D}_{0}=\mathrm{Control}(\mathrm{No}$ stress), $\mathrm{D}_{1}=$ Crown root initiation stage (20-19 DAS), $\mathrm{D}_{2}=$ Booting stage (45-54 DAS) and $\mathrm{D}_{3}=$ Anthesis stage (55-64 DAS)

Table 6. Interaction effects of organic manures and drought stress on yields and harvest index of wheat

\begin{tabular}{ccccc}
\hline $\begin{array}{c}\text { Interaction (Organic } \\
\text { manure } \times \text { Drought) }\end{array}$ & $\begin{array}{c}\text { Grain yield } \\
\text { plant }^{-1} \\
(\mathbf{g})\end{array}$ & $\begin{array}{c}\text { Straw yield } \\
\text { plant }^{-\mathbf{1}} \\
(\mathbf{g})\end{array}$ & $\begin{array}{c}\text { Biological }^{\mathbf{1}} \\
\text { yield plant }^{\mathbf{1}}\end{array}$ & $\begin{array}{c}\text { Harvest index } \\
(\mathbf{g})\end{array}$ \\
\hline $\mathrm{O}_{0} \mathrm{D}_{0}$ & $4.07 \mathrm{~cd}$ & $4.48 \mathrm{c}$ & $8.55 \mathrm{c}$ & $47.52 \mathrm{a}-\mathrm{d}$ \\
$\mathrm{O}_{0} \mathrm{D}_{1}$ & $2.78 \mathrm{fg}$ & $3.40 \mathrm{ef}$ & $6.18 \mathrm{ef}$ & $45.00 \mathrm{~d}-\mathrm{f}$ \\
$\mathrm{O}_{0} \mathrm{D}_{2}$ & $3.91 \mathrm{~d}$ & $3.86 \mathrm{~d}$ & $7.77 \mathrm{~d}$ & $50.32 \mathrm{a}$ \\
$\mathrm{O}_{0} \mathrm{D}_{3}$ & $2.47 \mathrm{~g}$ & $3.16 \mathrm{f}$ & $5.63 \mathrm{f}$ & $43.80 \mathrm{f}$ \\
$\mathrm{O}_{1} \mathrm{D}_{0}$ & $4.72 \mathrm{a}$ & $4.96 \mathrm{a}$ & $9.69 \mathrm{a}$ & $48.75 \mathrm{ab}$ \\
$\mathrm{O}_{1} \mathrm{D}_{1}$ & $4.00 \mathrm{~cd}$ & $4.41 \mathrm{c}$ & 8.41 & $47.47 \mathrm{a}-\mathrm{d}$ \\
$\mathrm{O}_{1} \mathrm{D}_{2}$ & $4.55 \mathrm{ab}$ & $4.87 \mathrm{ab}$ & $9.42 \mathrm{ab}$ & $47.10 \mathrm{~b}-\mathrm{e}$ \\
$\mathrm{O}_{1} \mathrm{D}_{3}$ & $2.96 \mathrm{f}$ & $3.51 \mathrm{e}$ & $6.47 \mathrm{e}$ & $45.72 \mathrm{c}-\mathrm{f}$ \\
$\mathrm{O}_{2} \mathrm{D}_{0}$ & $4.30 \mathrm{bc}$ & $4.65 \mathrm{a}-\mathrm{c}$ & $8.95 \mathrm{bc}$ & $48.02 \mathrm{a}-\mathrm{c}$ \\
$\mathrm{O}_{2} \mathrm{D}_{1}$ & $3.35 \mathrm{e}$ & $3.87 \mathrm{~d}$ & $7.22 \mathrm{~d}$ & $46.39 \mathrm{~b}-\mathrm{f}$ \\
$\mathrm{O}_{2} \mathrm{D}_{2}$ & $4.10 \mathrm{~cd}$ & $4.56 \mathrm{bc}$ & $8.66 \mathrm{c}$ & $47.38 \mathrm{a}-\mathrm{d}$ \\
$\mathrm{O}_{2} \mathrm{D}_{3}$ & $2.47 \mathrm{~g}$ & $3.12 \mathrm{f}$ & $5.59 \mathrm{f}$ & $44.24 \mathrm{ef}$ \\
\hline $\mathrm{SE}+$ & 0.16 & 0.15 & 0.30 & 1.43 \\
$\mathrm{CV}(\%)$ & 5.51 & 4.67 & 4.85 & 3.75 \\
\hline
\end{tabular}

In a column means having similar letter(s) are statistically similar and those having dissimilar letter(s) differ significantly at $5 \%$ level of significance

Here: $\mathrm{O}_{0}=$ Control (Only RDCF+ no organic manure), $\mathrm{O}_{1}=\mathrm{RDCF}+$ Cowdung, $\mathrm{O}_{2}=\mathrm{RDCF}+$ Poultry, $\mathrm{D}_{0}=\mathrm{Control}(\mathrm{No}$ stress), $\mathrm{D}_{1}=$ Crown root initiation stage (20-19 DAS), $\mathrm{D}_{2}=$ Booting stage (45-54 DAS) and $\mathrm{D}_{3}=$ Anthesis stage (55-64 DAS)

\section{Conclusion}

It may be concluded from the result that, application of cowdung $\left(10 \mathrm{t} \mathrm{ha}^{-1}\right)$ seems promising to overcome yield loss due to drought stress at booting stage of wheat.

Acknowledgement: Ministry of Science and Technology.

\section{References}

Akram, M. 2011. Growth and yield components of wheat under water stress of different growth stages. Bangladesh J. Agril. Res. 36(3): 455-468.

Alghabari, F. and M.Z. Ishan. 2018. Effect of drought stress on growth, grain filling duration, yield and quality attributes of barley (Houdium vulgare 1.). Bangladesh J. Bot. 47(3): 421-428.

BBS (Bangladesh Bureau of Statistics). 2018. 45 years Agriculture Statistics of Major Crops. Bangladesh Bureau of Statistics (BBS) Statistics and Informatics Division (SID), Ministry of Planning. p.194. 
Farooq, M., A. Wahid, N. Kobayashi, D. Fujita and S.M.A. Basra. 2009. Plant drought stress: effects, mechanisms and management. Agron. Sust. Dev. 29: 185-210.

Hammad, H.M., A. Khaliq, A. Ahmad and K. Laghar. 2011. Influence of different organic manures on wheat productivity. Intl. J. Agric. Biol. 13(1): 137-140.

Havaux, M. 1992. Stress tolerance of photo system II in vivo: Antagonistic effects of water, heat, and photo inhibition stresses. Plant Physiol. 100: 424-432.

Ibrahim, M., A. Hussain, M. Iqbal and E. Valeem. 2008. Response of wheat growth and yield to various levels of compost and organic manure. Pakistan J. Bot. 40(5): 2135-2141.

Mirzaei, A., R. Naseri, and R. Soleimani. 2011. Response of different growth stages of wheat to moisture tension in a semiarid land. World Appl. Sc. J. 12(1): 83-89.

Rajaram, S. 2000. International wheat breeding: Past and present achievements and future directions. In Warren E Kronstand Honorary Symposium, 18 Feb 1999, Oregon State University Extension Service, Special Report p.1017.

Sarwar, G. 2005. Use of compost for crop production in Pakistan. Ökologie und Umweltsicherung. 26/2005. Universität Kassel.

Uyanoz, R., U. Cetin and E. Karaarslan. 2006. Effect of Organic Materials on Yields and Nutrient Accumulation of Wheat. J. Plant Nut. 29(5): 959-974.

Yassen, A.A., M. Abd El-Hady and S.M. Zaghloul. 2006. Replacement part of mineral N fertilizer by organic ones and its effect on wheat plant under water regime conditions. World J. Agric. Sci. 2: 421-428.

Zhao, C.X., L.Y. Guo, C.A. Jaleel, H.B. Shao and H.B. Yang. 2008. Prospects for dissecting plant-adaptive molecular mechanisms to improve wheat cultivars in drought environments. Comp. Rend. Biol. 331: 579-586. 